\title{
Einführung in OSCE Insights 2020: Die OSZE im Krisenmodus
}

\author{
Cornelius Friesendorf
}

Bitte zitieren als: Cornelius Friesendorf, Einführung in OSCE Insights 2020: Die OSZE im Krisenmodus (Baden-Baden: Nomos, 2021), https://doi.org/10.5771/9783748911630-00

Dies ist der erste Jahrgang von OSCE Insights, der neuen Schriftenreihe des Zentrums für OSZE-Forschung (CORE), Institut für Friedensforschung und Sicherheitspolitik an der Universität Hamburg (IFSH). OSCE Insights ist die Nachfolgepublikation des OSZE-Jahrbuchs, das vom IFSH von 1995 bis 2019 herausgegeben wurde. Unsere neue Reihe untersucht alle drei OSZE-Dimensionen und Themen wie Konfliktmanagement, Rüstungskontrolle, Demokratisierung, Reform des Sicherheitssektors (SSG/R), Umweltschutz, Konnektivität und Menschenrechte. Autoren beschäftigen sich weiterhin mit institutionellen Veränderungen der OSZE und den Interessen und Strategien der Teilnehmerstaaten.

OSCE Insights-Autor*innen kommen aus der universitären und politiknahen Forschung wie auch aus der Praxis. Ein wesentliches Ziel ist es, Forschungsergebnisse für Entscheidungsträger*innen und Praktiker"innen zu „übersetzen“ und in

* Dr. habil. Cornelius Friesendorf

Institut für Friedensforschung und Sicherheitspolitik an der Universität Hamburg (IFSH)

friesendorf@ifsh.de
Form von Empfehlungen zu formulieren. So wollen wir zum Ziel der OSZE beitragen, umfassende und kooperative Sicherheit zu fördern.

Alle Beiträge erscheinen in englischer, russischer und deutscher Sprache und werden über das Jahr verteilt in der eLibrary des Nomos-Verlags sowie auf der Website des IFSH frei zugänglich gemacht. Darüber hinaus erscheinen alle Beiträge am Anfang eines neuen Jahres in Druckform, auch in den drei Sprachen. Eine anonyme Begutachtung und intensive redaktionelle Bearbeitung der Texte sollen sicherstellen, dass unsere Leser*innen zuverlässig und in einer unbürokratischen Sprache informiert werden.

In dieser ersten Ausgabe von OSCE Insights stehen Krisen im Mittelpunkt. Der Untertitel „Corona, Krieg, Führungskrise" verweist auf drei Krisen, von der die OSZE im Jahr 2020 betroffen war. Erstens beeinträchtigte die Coronapandemie das Tagesgeschäft der OSZE, die online arbeiten musste, obwohl persönliche Kontakte zentral für diese stark auf Informalität basierende Organisation sind. Zweitens eskalierte der Konflikt zwischen Armenien und Aserbaidschan über Bergkarabach erneut zu einem Krieg, der die 
Machtverteilung in der Region grundlegend veränderte. Unterdessen gab es in der Ostukraine weiterhin Tote und Verletzte. Drittens waren in der vielleicht dramatischsten institutionellen Krise in der Geschichte der OSZE die vier Führungspositionen des Sekretariats, des Büros für demokratische Institutionen und Menschenrechte, des Hohen Kommissars für nationale Minderheiten und des Beauftragten für Medienfreiheit monatelang unbesetzt, nachdem mehrere Teilnehmerstaaten aufgrund von nationalem Egoismus, Kritik durch OSZE-Institutionen oder Fehleinschätzungen die Verlängerung der Mandate der Amtsinhaber*innen nicht befürwortet hatten.

Die Beiträge in OSCE Insights 2020 analysieren verschiedene Arten von Krisen. Eine erste Gruppe von Beiträgen untersucht die Schwierigkeiten bei der Umsetzung von OSZE-Verpflichtungen und Versäumnisse bei der systematischen Nutzung der bestehenden OSZE-Instrumente.

Der erste Beitrag beschäftigt sich mit den Schwierigkeiten der OSZE, demokratische Polizeiarbeit in Zentralasien zu unterstützen. Die Regierungen dort wollen technische Hilfe bei der Strafverfolgung und unterstützen oder tolerieren auch Initiativen zur Verbesserung der menschlichen Sicherheit. Eine Kontrolle über die Polizei etwa durch die Zivilgesellschaft stößt bei den Regierungen in Zentralasien und auch in Russland aber auf Widerstand - schließlich bedrohen liberale Modelle der guten Regierungsführung Klientelismus. Die Anpassung der OSZE-Feldoperationen an die Prioritäten des Gastlandes steht im Einklang mit dem OSZE-
Prinzip von national ownership, birgt aber die Gefahr, den Autoritarismus zu verstärken.

Andrew Baker zeigt, dass die Umsetzung von OSZE-Verpflichtungen auch bei der Bekämpfung des Antisemitismus schwierig ist. Baker dokumentiert den unzureichenden Schutz für jüdische Gemeinden durch Teilnehmerstaaten, obwohl diese Gemeinden hohen Risiken ausgesetzt sind. Er verweist auf die gemischte Bilanz der OSZE bei der Verabschiedung und Umsetzung einer umfassenden Definition von Antisemitismus und zeigt, wie das Konsensprinzip und personelle Veränderungen in der OSZE die Fähigkeit der Organisation beeinträchtigt haben, rasch und entschieden gegen Antisemitismus vorzugehen.

Michael Raith erläutert, dass die OSZE über viele Instrumente zur Konfliktbewältigung verfügt, die sowohl für die Verhütung und Beilegung gewaltsamer Konflikte als auch für die Unterstützung von Staaten und Gesellschaften nach Beendigung der Kampfhandlungen wichtig sind. Allerdings hat die OSZE Schwierigkeiten, diese Instrumente systematisch zu nutzen, nicht zuletzt aufgrund von Unterfinanzierung und mangelndem Interesse seitens der Teilnehmerstaaten. Raiths Analyse legt nahe, dass selbst geringfügige zusätzliche Investitionen, wie etwa in die Aufstockung des Personals im „Situation Room“ des Konfliktverhütungszentrums, bei zentralen Aufgaben wie der Frühwarnung etwas bewirken könnten.

Sebastian Mayers Analyse der Ambitionen Kasachstans, ein OSZE-Konnektivitätszentrum im eigenen Land einzurichten, macht deutlich, in welchem Aus- 
maß Staaten OSZE-Verpflichtungen zunehmend infrage stellen, insbesondere in der menschlichen Dimension. Der von Kasachstan favorisierte à-la-carte-Ansatz wirft Fragen über die Zukunft der menschlichen Dimension und damit über das Konzept der umfassenden Sicherheit und der OSZE insgesamt auf. Die Forderungen Kasachstans deuten auf Machtverschiebungen im OSZE-Raum hin: Viele Teilnehmerstaaten akzeptieren die Rolle des Normnehmers nicht mehr, und die Unausweichlichkeit von Demokratisierung steht zunehmend in Frage.

Auch die Beiträge 5, 6 und 9 unterstreichen Herausforderungen für OSZEVerpflichtungen und zeigen, dass Teilnehmerstaaten das Potenzial der OSZE nicht voll ausschöpfen. Alexander Lambert, Filip Ejdus und Thomas Schmidt untersuchen innerstaatliche Einsätze von Streitkräften zur Bewältigung der Coronapandemie und nehmen den OSZE-Verhaltenskodex zu politisch-militärischen Aspekten der Sicherheit aus dem Jahr 1994 als Maßstab für die Angemessenheit solcher Einsätze. Während sich die Staaten meist an den Kodex gehalten haben, zeigen die Autoren auch, dass manche Verwendungen des Militärs Fragen etwa nach Notwendigkeit und VerhältnismäBigkeit aufwerfen.

Der Krieg zwischen Armenien und Aserbaidschan um Bergkarabach im Herbst 2020, den Philip Remler, Richard Giragosian, Marina Lorenzini und Sergej Rastoltsev analysieren, war ein Verstoß gegen ein zentrales OSZE-Prinzip: die friedliche Streitbeilegung. Überdies zeigen Remler et al., dass die Minsk-Gruppe der OSZE als primäres internationales
Verhandlungsformat marginalisiert wurde.

Frank Evers, André Härtel und Marietta König untersuchen die Zusammenarbeit zwischen der OSZE und dem Europarat. Die sich überschneidenden und ergänzenden Funktionen der beiden Organisationen und die Tatsache, dass beide direkt von der Krise des Multilateralismus betroffen sind, machen sie zu natürlichen Partnern. Allerdings finden Treffen auf höchster Ebene nur selten und zudem ritualisiert statt, und die Feldoperationen der beiden Organisationen kooperieren nicht systematisch miteinander.

Eine weitere Gruppe von Beiträgen erörtern konträre Positionen von Teilnehmerstaaten, die Bemühungen um Vertrauensbildung und friedliche Konfliktbeilegung behindert haben. Philip Remler et al. argumentieren, dass die Co-Vorsitzenden der Minsk-Gruppe nicht in der Lage waren, sich effektiv an Friedensverhandlungen zu beteiligen, weil die Repräsentanten Armeniens und Aserbaidschans unvereinbare, maximale Forderungen erhoben. Solche Maximalpositionen hatten auch die Bevölkerungen kompromissunwillig gemacht.

Benjamin Schaller erläutert, wie die Rüstungskontrollabteilungen diverser Staaten weiterhin vertrauens- und sicherheitsbildende Maßnahmen (VSBM) auf der Arbeitsebene umsetzen. Dennoch reichen diese positiven transnationalen Beziehungen nicht aus, um auf politischer Ebene Vertrauen zwischen Russland und westlichen Staaten aufzubauen.

Mit dem Fokus auf gesellschaftliche Narrative zum Krieg in der Ostukraine präsentieren Cécile Druey, Anna Hess, 
Julia Kaplan und Valentina Cherevatenko empirische Forschungsergebnisse, die für diejenigen ernüchternd sein dürften, die davon ausgehen, dass Politiker zwar den Krieg, Gesellschaften aber den Frieden suchen. Ihr Beitrag zeigt, dass die Positionen der Befragten in der Ukraine und in Russland zum Minsker Prozess und zur Schlüsselfrage, wie und ob die ukrainische Staatlichkeit in nichtstaatlich kontrollierten Gebieten wiederhergestellt werden soll, weitgehend mit den offiziellen Positionen der Konfliktparteien identisch sind.

Es gibt aber auch Grund zur Hoffnung. Die OSZE war schon immer ein Forum sehr unterschiedlicher Staaten. 2020 als das schlechteste Jahr in der Geschichte der Organisation zu bezeichnen lässt die vielen stürmischen Zeiten, die die OSZE seit der Charta von Paris 1990 überstanden hat, außer Betracht. Tatsächlich hat die OSZE im Jahr 2020 ihre Widerstandsfähigkeit bewiesen; und ihre Verpflichtungen sind, auch wenn sie oft verletzt werden, nach wie vor unverzichtbar für die Regelung des Verhaltens innerhalb und zwischen Staaten. Alle Autor"innen dieses Bandes zeigen Möglichkeiten für die OSZE auf.

Der erste Beitrag zeigt, dass die OSZE zentralasiatische Bevölkerungen unterstützen kann, auch ohne innerstaatliche Machtverhältnisse $\mathrm{zu}$ verändern. Die Analysen von Baker und Raith nennen mehr politische Unterstützung und Ressourcen als Voraussetzungen für eine bessere Einhaltung der OSZE-Verpflichtungen (Baker) und die Fähigkeit der OSZE zum Konfliktmanagement (Raith). Evers et al. formulieren Empfehlungen für engere Beziehungen zwischen der OSZE und dem Europarat, unter anderem durch mehr informelle Interaktion zwischen hochrangigen Vertreter*innen der beiden Organisationen. In Bezug auf Karabach argumentieren Remler et al., die Minsk-Gruppe könnte dabei helfen, VSBM zu schaffen, Verhandlungen über den künftigen Status des umstrittenen Gebiets zu unterstützen und auf ein regionales Friedensabkommen hinzuarbeiten. Schaller empfiehlt, VSBM sollten sich unter anderem mehr auf die multilaterale Verifikation und Vertrauensbildung auf der politisch-strategischen Ebene konzentrieren. Unter Anwendung der Verhandlungstheorie gehen Druey et al. vom Vergleich unterschiedlicher Positionen zur Identifizierung zugrunde liegender Interessen über. Letztere lassen Gemeinsamkeiten erkennen, die Raum bieten für eine nachhaltige und friedliche Lösung des Konflikts im Donbass.

In der Tat zeigte der Ministerrat 2020, dass die Teilnehmerstaaten ein Interesse daran haben, die OSZE am Leben zu erhalten. Regierungen verabschiedeten Beschlüsse zu Themen, über die ein relativer Konsens besteht, wie etwa die Bekämpfung der organisierten Kriminalität. Sie bekräftigten sogar die anhaltende Relevanz von Menschenrechtsnormen und -verpflichtungen, indem sie einen Beschluss gegen Folter fassten. Vor allem aber besetzten sie die vier Spitzenpositionen des Sekretariats und der Institutionen. Zugleich deuten jedoch interpretative Erklärungen, insbesondere der Vereinigten Staaten und Russlands, darauf hin, dass mächtige Teilnehmerstaaten sehr unterschiedliche Ansichten darüber vertre- 
ten, welche Kompetenzen und Prioritäten der OSZE-Apparat haben sollte.

Viele Menschen haben dazu beigetragen, diese erste Ausgabe von OSCE Insights unter den schwierigen Bedingungen des Jahres 2020 zu erstellen. Die Autor*innen investierten viel Zeit in das Verfassen und Überarbeiten der Texte, wobei sie manchmal mehrere Überarbeitungsrunden über sich ergehen lieBen. Externe Gutachter*innen reagierten schnell auf unsere Einladungen, Texte zu kommentieren, und passten ihre Bewertungskriterien an unser policy-Format an. Ein großer Dank geht auch an das OSCEInsights-Team - Carolyn Benson, Ursula Froese, Alona Shestopalova, Caroline Taylor - sowie an unsere Übersetzer*innen und Lektor*innen für die Bearbeitung der russischsprachigen und deutschsprachigen Ausgaben. Unterstützung erhielt das Team auch von anderen IFSHKolleg*innen, insbesondere von Frank Evers, Britta Fisch, Alexandra Harm, Sonja Objartel und Barbara Renne. Eva Lang und Martin Reichinger vom Nomos Verlag haben so manche Änderungen an den Korrekturfahnen in der letzten Minute toleriert und sind gleichzeitig unserem Wunsch entgegengekommen, die Beiträge so schnell wie möglich online zu stellen. Das Auswärtige Amt stellte großzügig Mittel sowie Ideen und Kontakte zur Verfügung. Ein besonderer Dank geht an Ursel Schlichting, die Ende 2020 das IFSH verließ. Über zwanzig Jahre lang sorgte sie dafür, dass das OSZE-Jahrbuch ein wichtiges Forum für OSZE-Debatten war. OSCE Insights hat ein solides Fundament. 
